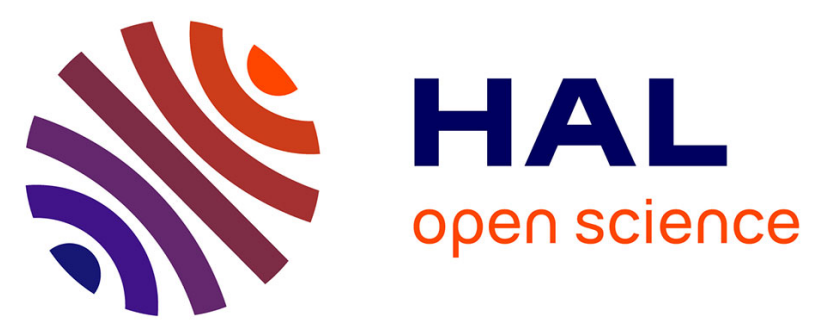

\title{
Targeting Both Viral and Host Determinants of Human Immunodeficiency Virus Entry, Using a New Lentiviral Vector Coexpressing the T20 Fusion Inhibitor and a Selective CCL5 Intrakine
}

\author{
Nicolas Petit, Karim Dorgham, Béatrice Levacher, Aude Burlion, Guy \\ Gorochov, Gilles Marodon
}

\section{To cite this version:}

Nicolas Petit, Karim Dorgham, Béatrice Levacher, Aude Burlion, Guy Gorochov, et al.. Targeting Both Viral and Host Determinants of Human Immunodeficiency Virus Entry, Using a New Lentiviral Vector Coexpressing the T20 Fusion Inhibitor and a Selective CCL5 Intrakine. Human gene therapy methods, 2014, 25 ((4)), pp.232-40. 10.1089/hgtb.2014.034 . inserm-01090571

\section{HAL Id: inserm-01090571 https://www.hal.inserm.fr/inserm-01090571}

Submitted on 3 Dec 2014

HAL is a multi-disciplinary open access archive for the deposit and dissemination of scientific research documents, whether they are published or not. The documents may come from teaching and research institutions in France or abroad, or from public or private research centers.
L'archive ouverte pluridisciplinaire HAL, est destinée au dépôt et à la diffusion de documents scientifiques de niveau recherche, publiés ou non, émanant des établissements d'enseignement et de recherche français ou étrangers, des laboratoires publics ou privés. 


\title{
Targeting Both Viral and Host Determinants of Human Immunodeficiency Virus Entry, Using a New Lentiviral Vector Coexpressing the T20 Fusion Inhibitor and a Selective CCL5 Intrakine
}

\author{
Nicolas Petit, ${ }^{1-3}$ Karim Dorgham, ${ }^{1-3}$ Béatrice Levacher, ${ }^{4}$ Aude Burlion, ${ }^{1-3}$ \\ Guy Gorochov, ${ }^{1,2,5, *}$ and Gilles Marodon ${ }^{1-3, *}$
}

\begin{abstract}
Numerous strategies targeting early and late steps of the HIV life cycle have been proposed for gene therapy. However, targeting viral and host determinants of HIV entry is the only strategy that would prevent viral DNAmediated $\mathrm{CD}^{+}{ }^{+}$cell death while diminishing the possibility for the virus to escape. To this end, we devised a bicistronic lentiviral vector expressing the membrane-bound form of the T20 fusion inhibitor, referred to as the C46 peptide, and a CCR5 superagonist, modified to sequester CCR5 away from the cell surface, referred to as the P2-CCL5 intrakine. We tested the effects of the vector on HIV infection and replication, using the human CEMR5 cell line expressing CD4 and CCR5, and primary human T cells. Transduced cells expressed the C46 peptide, detected with the 2F5 monoclonal antibody by flow cytometry. Expression of the P2-CCL5 intrakine correlates with lower levels of cell surface CCR5. Complete protection against HIV infection could be observed in cells expressing the protective transgenes. Importantly, we show that the combination of the transgenes was more potent than either transgene alone, showing the interest of expressing two entry inhibitors to inhibit HIV infection. Last, genetically modified cells possessed a selective advantage over nonmodified cells on HIV challenge in vitro, showing that modified cells were protected from HIV-induced cell death. Our results demonstrate that lentiviral vectors coexpressing the T20 fusion inhibitor and the P2-CCL5 intrakine represent promising tools for HIV gene therapy.
\end{abstract}

\section{Introduction}

$\mathbf{H}$ UMAN IMMUNODEFICIENCY VIRUS entry involves sequential binding, first with the CD4 receptor and next with a chemokine coreceptor, CCR5 or CXCR4. A 32-bp deletion in the $C C R 5$ gene $(\Delta 32)$ confers resistance to HIV infection in homozygotes (Deng et al., 1996). A hematopoietic stem cell (HSC) transplant for acute myeloid leukemia (AML) in an HIV-infected patient was performed, using allogeneic cells from a CCR5 $\Delta 32$ donor (Hutter et al., 2009). This study showed for the first time that a cure for HIV infection was possible and further highlighted the interest of targeting CCR5 through a gene therapy approach (Allers et al., 2011).
Various strategies have been proposed to disrupt CCR5 expression: one targets directly the CCR5 gene with zinc finger nucleases (ZFNs) and induces stable deletions in the CCR5 gene that prevent expression of the molecule at the cell surface (Perez et al., 2008). This has been shown to prevent $\mathrm{CD}^{+}{ }^{+} \mathrm{T}$ cell depletion, but not viral replication, in humanized mice (Holt et al., 2010). This strategy is currently being tested in a phase 1 clinical trial using genemodified $\mathrm{T}$ cell transfer (Tebas et al., 2014). Another approach relies on CCR5-specific short hairpin RNA (shRNA) (Qin et al., 2003). A significant effect on HIV replication of a CCR5-specific shRNA was observed if combined with TRIM5 $\alpha$ (tripartite motif-5 $\alpha$ ) and trans-activation response

\footnotetext{
${ }^{1}$ Sorbonne Universités, UPMC Univ Paris 06, CR7, Centre d'Immunologie et des Maladies Infectieuses (CIMI), F-75013, Paris, France. ${ }^{2}$ INSERM, U1135 (CIMI), Paris F-75013, France.

${ }^{3}$ CNRS, ERL 8255 (CIMI), Paris F-75013, France.

${ }^{4}$ INSERM U959, Paris F-75013, France.

${ }^{5}$ Service Immunologie, Hôpital Pitié-Salpétrière, AP-HP, Paris F-75013, France.

*Joint co-authorship.
} 
(TAR) element decoy genes (Walker et al., 2012). However, a clinical trial to determine safety, using a similar strategy targeting early and late steps of the viral life cycle, failed to show any improvements in immunological or virological parameters (DiGiusto et al., 2010). Thus, although effective in vitro, in vivo results, either in animal models or clinical trials, failed to fulfill expectations. Additional HIV gene therapy strategies and tools must therefore be devised and tested.

Apart from genetic knockouts, another possibility to modulate CCR5 at the cell surface is to target the CCR5 protein by intracellular antibody (Swan et al., 2006) or intracellular chemokine (intrakine) gene delivery (Yang et al., 1997). These strategies reduce CCR5 at the cell surface but do not completely abolish expression. Molecular evolution of CCL5 in vitro led to selection of the P2-CCL5 polypeptide, endowed with higher affinity and selectivity for CCR5 than parental CCL5. The soluble P2-CCL5 compound is a potent inhibitor of HIV entry in vitro (Hartley et al., 2003; Jin et al., 2010). We engineered a P2-CCL5 intrakine bearing a KDEL sequence that allows its specific compartmentalization into the granular endoplasmic reticulum (GER). Sequestration of the protein into the GER should reduce CCR5 expression from the cell surface and thus directly impact the sensitivity of modified cells to HIV infection.

The C46 peptide is derived from the clinically approved gp41-analog peptide fusion inhibitor T20 (enfuvirtide) (Hildinger et al., 2001). Primary human $\mathrm{CD}^{+} \mathrm{T}$ cells modified by a self-inactivating lentiviral vector encoding the C46 peptide were protected from HIV infection in vitro (Egelhofer et al., 2004; Perez et al., 2005). Furthermore, a synergistic effect of T20 and low levels of CCR5 was demonstrated (Heredia et al., 2007). We thus reasoned that coexpression of the P2-CCL5 intrakine with the C46 entry inhibitor should lead to better protection against HIV infection. In the present study, we developed a lentiviral vector combining the C46 peptide with the P2-CCL5 intrakine to improve antiviral efficacy and reduce the likelihood for escape variants to emerge. Our results show that genetically modified cells were protected from HIV infection and thus were conferred a selective advantage in vitro over nontransduced cells. Our results confirm that targeting viral and host determinants represents a promising strategy for HIV gene therapy.

\section{Materials and Methods}

\section{Cell culture}

HEK-293T/17 cells (CRL-11268; American Type Culture Collection [ATCC], Manassas, VA) were grown in Dulbecco's modified Eagle's medium (DMEM) supplemented with $2 \mathrm{~m} M$ L-glutamine, penicillin (100 U/ml), streptomycin $(100 \mu \mathrm{g} / \mathrm{ml})$ (Life Technologies, Cergy-Pontoise, France), and $10 \%$ heat-inactivated fetal calf serum (FCS). CEM.NKRCCR5 cells (CEMR5, cat. no. 4376; obtained from the AIDS Research Reagent Program, Germantown, MD) were cultured in RPMI 1640 supplemented with 10\% FCS and antibiotics. Primary human cells were obtained from leukapheresis samples collected from healthy donors after informed consent had been obtained. Cells were grown at a concentration of $1 \times 10^{6} \mathrm{cells} / \mathrm{ml}$ and activated in RPMI, FCS, antibiotics, interleukin-2 (Proleukin, $60 \mathrm{IU} / \mathrm{ml}$; Novartis, Basel, Switzerland), and CD3/CD28 beads (CTS Dynabeads CD3/CD28; Invitrogen, Carlsbad, CA) at 3 beads per cell. Cells were transduced with the indicated lentiviral vectors 3 days after activation and infected with HIV 3-5 days after transduction.

\section{Lentiviral vector design and production}

Second-generation self-inactivating (SIN) lentiviral vectors were used in this study. The cassette encoding the C46 peptide was kindly provided by D. von Laer (Innsbruck University, Innsbruck, Austria) and was cloned into pRRLsin.cPPT.hPGK (kind gift from Dr. L. Naldini, TIGET, San Raffaele Telethon Institute for Gene Therapy, San Raffaele Scientific Institute, Milan, Italy) to generate the Lv93 vector. The P2-CCL5 intrakine was constructed by PCR from the previously described phagemid vector (Hartley et al., 2003). The P2-CCL5 analog was linked at the $\mathrm{N}$ terminus with the CCL5 leader sequence, using an NcoItailed forward primer, and at the $\mathrm{C}$ terminus with an $\mathrm{HA}$ tag (YPYDVPDYA) and an endoplasmic reticulum (ER) retention signal (KDEL), using an XhoI-tailed reverse primer. The resulting P2-CCL5 intrakine (P2i) was further cloned into the lentiviral vector pRRLsin.PPT.hPGK to generate the Lv114 vector. The C46 coding sequence consists of the T20 sequence fused with the CD34 membrane-spanning domain separated by the human IgG2 hinge (Hildinger et al., 2001). The C46-2A-P2i bicistronic sequence was synthesized (GenScript, Piscataway, NJ) and inserted into the BamHI/ SalI sites in pRRLsin.PPT.hPGK or into the pRRLsin.PPT. EF1 $\alpha$ plasmid (described in Dupuy et al., 2005) to generate the Lv163 and Lv203 vectors, respectively. Details on the cloning procedures are available on request. Lentiviral vectors were produced in HEK-293T cells, as described (Marodon et al., 2003). Briefly, $23.3 \mu \mathrm{g}$ of the $\Delta 8.9$ packaging plasmid, $30 \mu \mathrm{g}$ of the transgene plasmid, and $10 \mu \mathrm{g}$ of the vesicular stomatitis virus (VSV)-G envelope were transfected into $15 \times 10^{6}$ cells in T-175 flasks by calcium phosphate precipitation. Vector supernatants were collected $48 \mathrm{hr}$ posttransfection and concentrated by ultrafiltration (Centricon Plus-70; Millipore, Molsheim, France) at $3500 \times g$ at $4^{\circ} \mathrm{C}$. Viral stocks were kept frozen at $-80^{\circ} \mathrm{C}$. Viral titers were determined on HEK-293T cells with various concentrations of vector supernatants in the presence of Polybrene ( $8 \mu \mathrm{g} / \mathrm{ml}$; Sigma-Aldrich, Saint-QuentinFallavier, France). Seventy-two hours after transduction, the percentage of cells expressing the transgenes was determined by flow cytometry and used to calculate a viral titer as the number of infectious particles per milliliter. Transductions of cells were performed with cell numbers and multiplicities of infections (MOIs) as indicated in the figure legends in the presence of Polybrene $(8 \mu \mathrm{g} / \mathrm{ml}$; SigmaAldrich).

\section{HIV strains}

HIV-1 molecular clones NL4D8 and Yu2 were obtained through the AIDS Research and Reference Reagent Program (gift from A. Moris, Centre Immunologie et Maladies Infectieuses, Paris, France). HIV stocks were prepared with $30 \mu \mathrm{g}$ of HIV plasmid transfected into $15 \times 10^{6} 293 \mathrm{~T}$ cells in T-175 flasks by calcium phosphate 
precipitation. Supernatants were collected $48 \mathrm{hr}$ posttransfection and frozen at $-80^{\circ} \mathrm{C}$. Viral titers $(\mathrm{ng} / \mathrm{ml})$ were determined by p24 ELISA with standard and unknowns measured in duplicate (Retrotek; ZeptoMetrix, Buffalo, NY).

\section{Flow cytometry and cell sorting}

Cells were washed twice in $1 \times$ phosphate-buffered saline (PBS) and stained with the cell viability dye eFluor 780 (eBioscience, San Diego, CA). The following monoclonal antibodies were used for cell surface staining: antiCCR5 conjugated with allophycocyanin (APC) (2D7; BD Biosciences, Pont-de-Claix, France), anti-CD4 conjugated with peridinin chlorophyll protein complex (PerCP) (RPAT-4; BioLegend/Ozyme, Saint-Quentin-en-Yvelines, France), and the human IgG1 2F5 monoclonal antibody $(\mathrm{mAb})$ directed to the EDLKWA gp41 epitope (Polymun, Klosterneuburg, Austria). Cells were washed and stained with a phycoerythrin (PE)-conjugated goat anti-human IgG antibody (Caltag/Life Technologies [Burlingame, CA] or Jackson ImmunoResearch [West Grove, PA]) to reveal 2F5 binding. For detection of the P2-CCL5 intrakine, cells were treated with brefeldin A for $2 \mathrm{hr}$, washed, fixed, and permeabilized for $20 \mathrm{~min}$ with an intracellular fixation \& permeabilization buffer kit (eBioscience). Cells were stained with biotinylated anti-CCL5 mAb (R\&D Systems, Lille, France) and revealed with streptavidin conjugated with PE-Cy7. All samples were acquired on an LSR II flow cytometer (BD Biosciences). Frequencies and median of fluorescence intensity (MFI) of positive cells were analyzed with FlowJo software (Tree Star, San Carlos, CA). Positivity was set according to the fluorescence minus one (FMO) control, in which staining incorporating all antibodies minus the one of interest are compared with staining with a complete set of antibodies. For cell-sorting experiments, Lv163transduced CEM-R5 cells were stained with 2F5 and PE-labeled secondary antibody 21 days after transduction. Cell sorting of $2 \mathrm{~F} 5^{\text {bright }}$ cells was performed on a FACSAria cell sorter (BD Biosciences).

\section{DNA extraction and HIV PCR}

We used a PureLink genomic DNA mini kit (Invitrogen, Saint Aubin, France) for DNA extraction. To detect HIV in a semiquantitative fashion, the SK38 and SK39 primers were used to detect gag viral DNA, and HLA-DQ-specific DQ28 and DQ29 were used to quantify total cellular DNA in the same tube, as described (Marodon et al., 1999). PCR conditions were as follows: 1 cycle at $94^{\circ} \mathrm{C}$ for $3 \mathrm{~min}$; followed by 30 cycles at $94^{\circ} \mathrm{C}$ for $30 \mathrm{sec}, 60^{\circ} \mathrm{C}$ for $30 \mathrm{sec}$, and $72^{\circ} \mathrm{C}$ for $30 \mathrm{sec}$; with a final extension cycle at $72^{\circ} \mathrm{C}$ for $10 \mathrm{~min}$. Fractions $(15 \mu \mathrm{l})$ of each PCR were run on a $4 \%$ agarose gel (Invitrogen) and analyzed on Quantum ST4 camera for gel electrophoresis (Vilber Lourmat, Marne-laVallée, France).

\section{Statistical analysis}

Two-tailed $p$ values were calculated with Prism version 6.0 software (GraphPad Software, San Diego, CA) for the Mac, using a parametric unpaired $t$ test with a confidence interval of $95 \%$.

\section{Results \\ Lentiviral vector designed to interfere with both viral and host determinants of HIV entry}

A series of mono- and bicistronic second-generation selfinactivated lentiviral vectors was constructed (Fig. 1A). Notably, we generated the Lv163 bicistronic lentiviral vector coexpressing the C46 peptide and the P2-CCL5 intrakine $(\mathrm{P} 2 \mathrm{i})$ under the control of the phosphoglycerate kinase (PGK) promoter (Fig. 1A). In our vectors, therapeutic cassette expression can be directly monitored by flow cytometry, using the 2F5 antibody, which binds to the C46 peptide (Hildinger et al., 2001). To facilitate the initial studies, we sorted Lv163-transduced CEMR5 cells on the basis of 2F5 staining by fluorescence-activated cell sorting (FACS) to generate CEMR5 cells expressing high and stable levels of C46 (CEMR5-163 cells) (Fig. 1B). CEMR5-163 cells expressed higher intracellular levels of CCL5 than did parental CEMR5 cells (Fig. 1B), showing that staining for CCL5 detected P2i as well. A lower level of expression of CCR5 was observed at the cell surface of CEMR5-163 cells compared with parental CEMR5 cells, showing that expression of intracellular $\mathrm{P} 2 \mathrm{i}$ correlated with reduced cell surface CCR5 expression (Fig. 1B).

\section{Bicistronic lentiviral vectors expressing C46 fusion inhibitor and CCL5 intrakine prevent HIV infection}

We next determined whether the high-level C46 expression and low-level CCR5 expression that we observed on the CEMR5-163 cell line described previously would have any impact on HIV infection. For this, we challenged CEMR5-163 or control CEMR5 cells with two different strains of HIV (the R5-tropic NL4D8 and Yu2 strains) and monitored HIV infection overtime by p24 ELISA in the supernatant and by HIV gag-specific DNA PCR at the end of the culture (Fig. 2). Control cells exhibited high levels of p24 (up to $1 \times 10^{5} \mathrm{pg} / \mathrm{ml}$ of supernatant from day 7 to day 11 postinfection) irrespective of the viral strain. Undetectable or low levels of p24 were measured in CEMR5-163 cells infected with NL4D8 virus (50 ng of p24), showing that expression of the transgenes had a protective effect at this dose of p24. This was confirmed by the lack of detectable provirus in challenged cultures (Fig. 2B), showing that expression of the transgenes provided complete protection. At the same dose, the $\mathrm{Yu} 2$ strain was more aggressive because CEMR5 control cells were all dead by day 10 from cytopathic effect (data not shown). Compared with controls, there was some viral replication detected in the culture infected with the $\mathrm{Yu} 2$ virus at $50 \mathrm{ng}$ of p24, as evidenced by p24 detection on day 7 and day 11 postinfection (Fig. 2C). However, CEMR5-163 cells were entirely protected from viral infection if infectious doses of $\mathrm{Yu} 2$ were reduced to $0.5 \mathrm{ng}$ of $\mathrm{p} 24$, whereas HIV was still detectable and highly pathogenic in control cells at this dose (Fig. 2D). Low p24 production in transduced cells could be due to increase cell death after HIV challenge. To the contrary, analysis with the viability dye included in our staining showed that the number of live cells dropped only in nontransduced cells, probably owing to HIV-related cell death (data not shown). Altogether, these results demonstrate that the lentiviral vector Lv163 protected genetically modified cells from infection. 
FIG. 1. A lentiviral vector designed to interfere with both viral and host determinants of HIV entry. (A) Schematic representation of the structure of the lentiviral vectors used in this study. LTR, long terminal repeat; cPPT, central polypurine tract of HIV; PGK, phosphoglycerate kinase promoter; T20m, membrane-bound form of T20 (C46 peptide); 2A, 2A sequence of the foot-and-mouth disease virus; P2i, P2-CCL5 intrakine (CCR5 superagonist); WPRE, woodchuck hepatitis virus regulatory element; $\triangle$ LTR, U3-deleted LTR. Indicated is the reference of the vector used throughout the study. Diagram is not to scale. (B) Expression of C46 peptide detected by 2F5 antibody in nontransduced cells (CEMR5NT) and CEMR5 cells after transduction with the Lv163 vector and sorting (CEMR5163). Percentages of positive cells are indicated on the profiles. (C) CCL5 expression and CCR5 expression in CEMR5 cells were assessed separately. The numbers above the profiles represent the median of fluorescence intensity (MFI) of the reported molecules according to the experimental conditions indicated in the legends (CEMR5-NT, nontransduced; Isotype, isotype control stained cells). Color images available online at www .liebertpub.com/hgtb

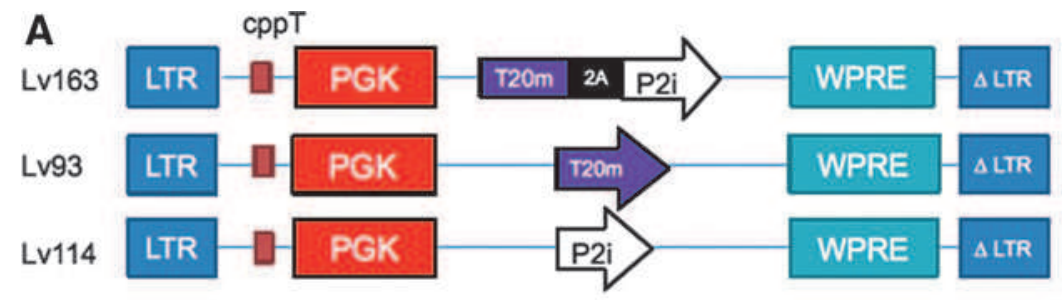

B
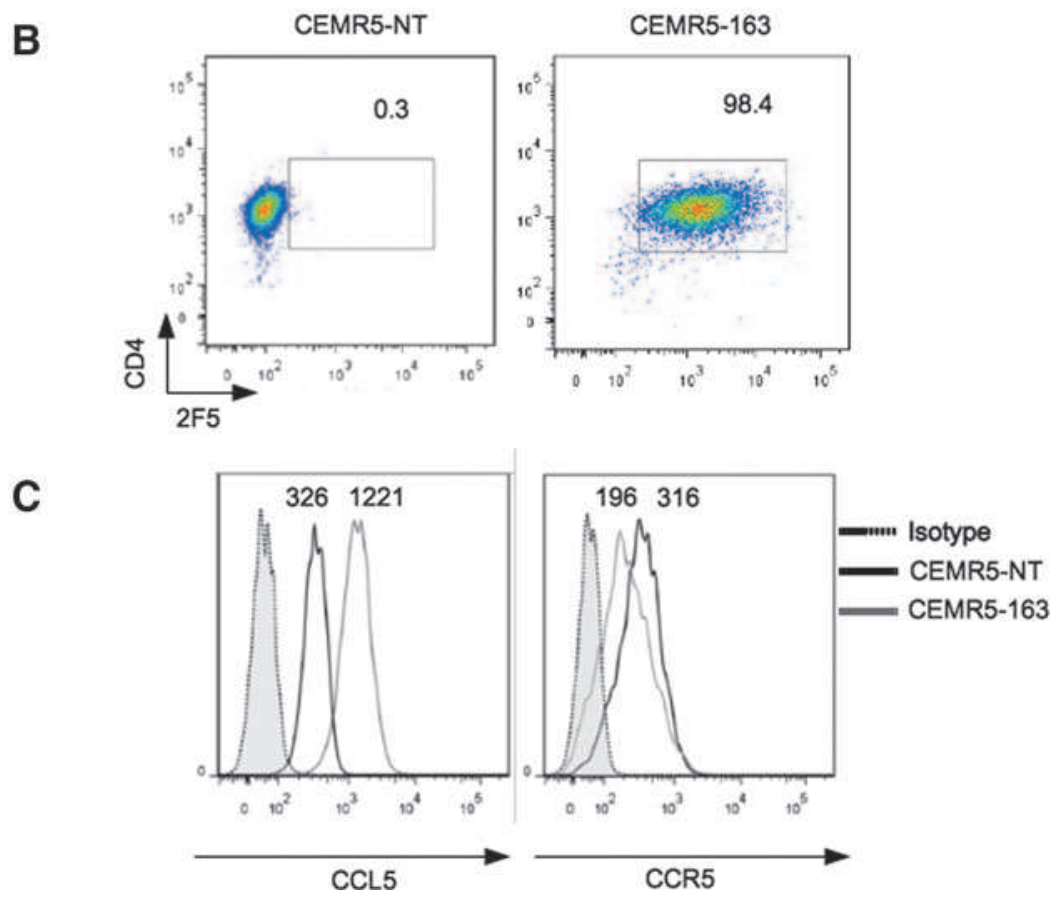

C46 fusion inhibitor and P2-CCL5 intrakine act in synergy to inhibit HIV infection

transgenes in the inhibition of HIV infection. For this, we sorted CEMR5 cells expressing low levels of CCR5 after transfection with the Lv114 plasmid (CEMR5-114 in Fig. 3A).

To document the advantage of coexpressing the P2-CCL5 intrakine and the C46 peptide, we tested the synergy of the

Low levels of CCR5 did not confer protection against HIV challenge, as monitored by p24 and gag detection (Fig. 3B and

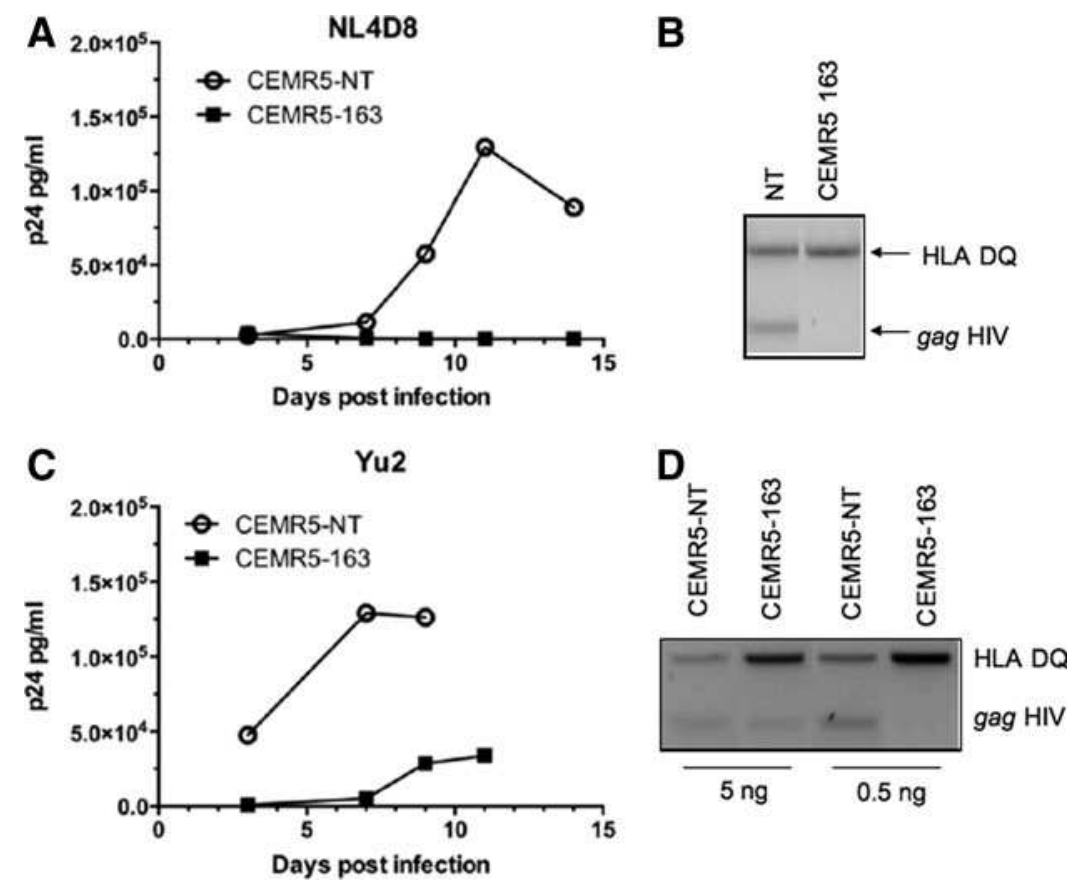

FIG. 2. Bicistronic lentiviral vectors expressing C46 fusion inhibitor and CCL5 intrakine prevent HIV infection. (A) CEMR5163 cells $\left(3 \times 10^{5}\right)$ were challenged with NL4D8 HIV (50 ng of p24). Viral replication is indicated by $\mathrm{p} 24$ detection in nontransduced cells (CEMR5-NT) and CEMR5-163 cells, as described in text. (B) PCR detection of HIV gag and HLA-DQ DNA on day 14 after infection under the indicated conditions. (C) CEMR5-163 cells or CEMR5-NT cells (nontransduced) $\left(3 \times 10^{5}\right)$ were challenged with Yu2 virus (50 ng of p24) and viral replication was monitored by p24 ELISA. (D) PCRs specific for HIV gag and HLA-DQ DNA were performed 24 days after infection with decreasing doses of HIV Yu2 as indicated. Results shown in (A-C) are from the same experiment whereas the PCR shown in (D) originated from an independent experiment. 
A
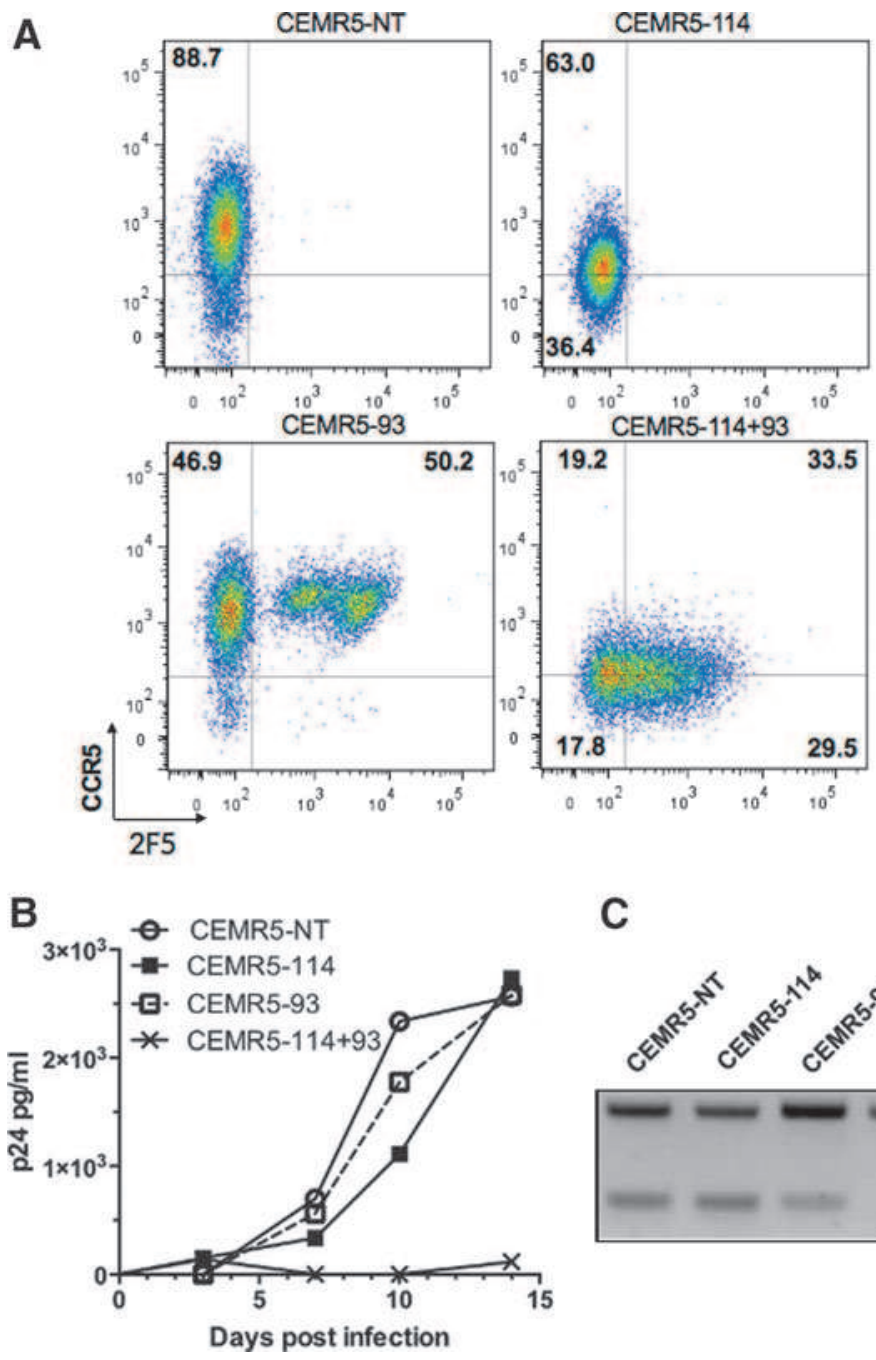

C

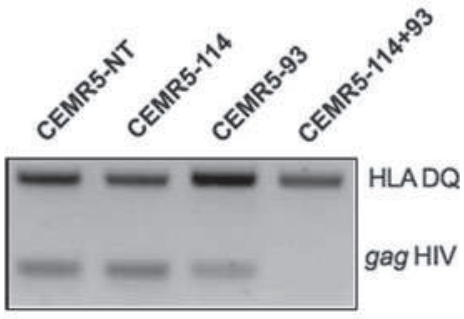

FIG. 3. The C46 fusion inhibitor and the P2-CCL5 intrakine act in synergy to inhibit HIV infection. (A) Fluorescence-activated cell-sorting (FACS) profile showing expression of the C46 peptide and CCR5 in control CEMR5 cells (top left panel) or after transfection with the Lv114 plasmid and sorted on the basis of CCR5 expression (CEMR5-114 cells, top right panel). FACS profile showing expression of $\mathrm{C} 46$ and CCR5 in CEMR5 cells (bottom left panel) and CEMR5-114 cells (bottom right panel) after transduction with the Lv93 vector. Percentages are indicated on the profiles for each quadrant. (B) p24 production in CEMR5 cells in the indicated culture conditions. CEMR5-NT (non transduced), CEMR5-114 (CCR5 $^{\text {lo }}$ cells), CEMR5$114+93\left(\right.$ CCR $5^{\text {lo }}$ cells + transduction with the Lv93 vector) CEMR5-93: parental CEMR5 cells tranduced by the Lv93 vector. (C) HIV gag and HLA-DQ specific DNA PCR were performed at the end of the indicated cultures. Color images available online at www.liebertpub.com/hgtb
C). However, transduction of these CEMR5-114 cells with the monocistronic lentiviral vector Lv93 (CEMR5-114+93 in Fig. 3A) led to complete protection, whereas cultures of CEMR5 cells containing $50 \%$ of $\mathrm{C} 46^{+}$cells (CEMR5-93 in Fig. 3A) were not protected (Fig. 3B). Thus, low CCR5 expression and $50-60 \%$ of C46 expression, two conditions in which cells were not protected, conferred protection to HIV infection if combined together.

\section{Selective advantage for CEMR5 cells expressing C46 fusion inhibitor and P2-CCL5 intrakine}

Our results so far demonstrate that the combination of two viral entry inhibitors could prevent HIV infection. We next asked whether this protection could prevent HIV-induced cell death and thus lead to better growth compared with nonprotected cells, a process that we referred to as a selective advantage. For that, gene-modified CEMR5 cells were infected or not with the R5-tropic HIV strain NL4D8 or Yu2, and the frequencies of $2 \mathrm{~F}^{+}$cells and the median of fluorescence intensity (MFI) of CCR5 in $2 \mathrm{~F}^{+}$cells were determined at the end of culture. A significant increase in the frequencies of $2 \mathrm{~F}^{+}$cells was observed when HIV-infected cultures were compared with noninfected ones (Fig. 4A). Overall, there was a 2.1-fold increase in the proportions of $2 \mathrm{~F}^{+}$cells in cultures in the presence of HIV (Fig. 4B). We assessed whether $2 \mathrm{~F}^{+}$ cells would also be enriched in $\mathrm{CCR} 5^{\text {lo }}$ cells, as expected from the action of the P2-CCL5 intrakine. Indeed, the MFI of CCR5 was lower on $2 \mathrm{~F}^{+}$cells in cultures infected with HIV compared with noninfected cultures (Fig. 4C and D). Altogether, these results demonstrate that $2 \mathrm{~F} 5{ }^{+} \mathrm{CCR} 5{ }^{\text {lo }} \mathrm{CEMR} 5$ cells were enriched on HIV infection in vitro. These results converge to show that expression of the therapeutic transgenes conferred a selective advantage to gene-modified cells.

\section{Expression of C46 peptide and of P2-CCL5 intrakine efficiently protects primary human $C D 4^{+} T$ cells from infection}

To determine whether the therapeutic transgenes would have similar effects on primary human $\mathrm{CD} 4^{+} \mathrm{T}$ cells, the natural target for HIV, we transduced activated peripheral blood mononuclear cells (PBMCs) with a new lentiviral vector in which the elongation factor- $1 \alpha(\mathrm{EF} 1 \alpha)$ promoter replaces the PGK promoter (Fig. 5A). Cultures were then challenged with two different HIV strains at two different doses. Transduction of PBMCs at an MOI of 40 led to reproducible expression of the C46 peptide in $60-80 \%$ of the cells 
FIG. 4. Selective advantage for CEMR5 cells expressing $\mathrm{C} 46$ fusion inhibitor and $\mathrm{P} 2$ CCL5 intrakine. (A) Frequencies of $2 \mathrm{~F} 5^{+}$ cells 10 days with (+ HIV) or without (-HIV) challenge with NL4D8 HIV (50 ng of p24). Percentages of $2 \mathrm{~F}^{+}$cells are indicated on the graph. (B) CEMR5 cells transduced with the Lv163 vector were challenged with NL4D8 HIV (10 or $50 \mathrm{ng}$ of p24). Results represents the frequencies of $2 \mathrm{~F}^{+}$cells in $\mathrm{CD}^{+}$cells (dead cells excluded) determined by flow cytometry from day 10 to day 14 after infection. Results are compiled from two independent experiments (n.s., not significant). (C) Median of fluorescence intensity (MFI) of CCR5 in $2 \mathrm{~F}^{+}$cells 10 days after challenge with $(+\mathrm{HIV})$ or without $(-\mathrm{HIV})$ NL4D8 virus (50 ng of p24). Numbers on the graph indicate the MFI of CCR5 in live $\mathrm{CD} 4{ }^{+} 2 \mathrm{~F} 5^{+}$cells for each histogram. (D) MFI of CCR5 in live $\mathrm{CD} 4^{+} 2 \mathrm{~F} 5^{+}$CEMR5 cells transduced with the Lv163 vector 10 days after challenge $(+\mathrm{HIV})$ or not $(-\mathrm{HIV})$ with NL4D8 or Yu2 virus (50 ng of p24) $(* p<0.05)$.

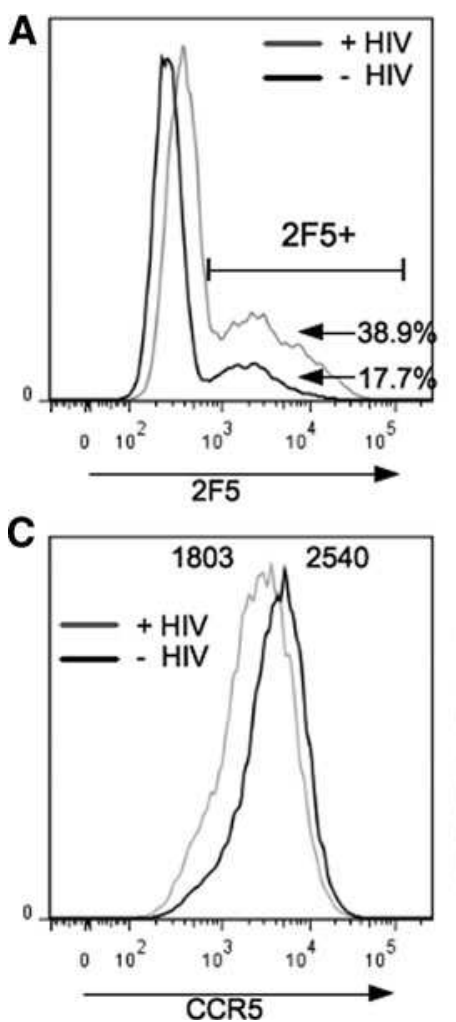

B
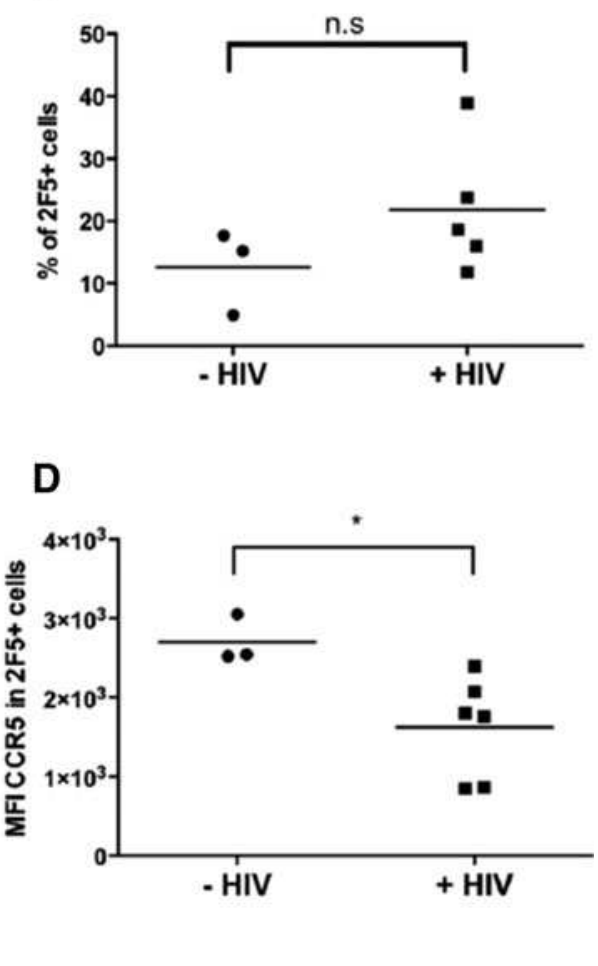

(Fig. 5B). Expression of P2-CCL5 was not monitored because activated human $\mathrm{CD}^{+}{ }^{+} \mathrm{T}$ cells express high levels of CCL5, rendering difficult the detection of the vectorencoded CCL5 by flow cytometry (data not shown). However, prevention of CCR5 expression at the surface of $2 \mathrm{~F}^{+}$cells was readily observed, indirectly showing that the P2-CCL5 intrakine affected CCR5 expression in primary $\mathrm{T}$ cells (Fig. 5C). A marked difference in p24 was observed when protected cultures were compared with mock-transduced cells, showing that expression of the transgenes affected viral replication (Fig. 5D). Furthermore, under conditions in which the number of $2 \mathrm{~F}^{+}$cells was set to lower frequencies by diluting transduced into nontransduced cells, a progressive increase in $2 \mathrm{~F} 5^{+}$cells was observed in HIV-infected relative to noninfected cultures, showing that transduced cells possessed a selective advantage over nonprotected cells (Fig. 5E).

\section{Discussion}

We devised a lentiviral vector expressing a combination of two HIV entry inhibitors to reduce the risks of viral escape and improve the likelihood for selective advantage. The combination of two entry inhibitors might be particularly interesting in view of data showing that the mere presence of viral DNA before integration might be the initiator of $\mathrm{CD}^{+}$ $\mathrm{T}$ cell death, the initial event in the development of AIDS (Doitsh et al., 2014). Thus, we believe that gene therapy and vaccines should aim toward the same goal, that is, preventing the virus from getting into the $\mathrm{CD} 4{ }^{+} \mathrm{T}$ cell.

HIV gene therapy has moved from single agents to combinatorial approaches targeting various steps of the viral cycle (Peterson et al., 2013). For instance, a lentiviral vector including a CCR5 shRNA, a chimeric TRIM5 $\alpha$ molecule, and a TAR decoy has been developed (Anderson et al., 2007) and promising results were published in humanized mice although the strategy failed to impact viral loads (Walker et al., 2012). However, the functional impact of overexpressing TRIM5 $\alpha$, APOBEC (apolipoprotein B mRNA-editing enzyme, catalytic polypeptide-like), or other mRNA-editing molecules has not been tested. In the vector described here, we took care not to incorporate mRNAediting molecules, or potential immunogens or proinflammatory molecules, that might prevent gene transfer efficacy or lead to unforeseeable side effects. Indeed, several reports attest that small interfering RNAs (siRNAs) might initiate a proinflammatory response in vivo (Judge et al., 2005; Robbins et al., 2008). Zinc finger nucleases might induce a complete loss of CCR5 and/or CXCR4 expression in targeted cells (Holt et al., 2010; Didigu et al., 2014) but the side effects of that protocol, such as off-target cleavage (Pattanayak et al., 2011), alteration of hematopoiesis, or homing properties for modified cells, still need to be fully evaluated. We chose a less aggressive strategy allowing residual expression of CCR5 on the cell surface. A partial downregulation of CCR5 expression alone might not be enough to completely protect $\mathrm{CD}^{+}{ }^{+} \mathrm{T}$ cells from infection but we show that it significantly increased the potency of the C46 peptide fusion inhibitor. This observation suggests that complete knockout of CCR5 might not be needed if modulation of CCR5 is combined with other therapeutic transgenes. Furthermore, the P2-CCL5 intrakine remains closely related to the self-derived CCL5, and is therefore unlikely to elicit a strong immune response. The $\mathrm{C} 46$ peptide is also a good candidate for gene therapy because it is derived from an already approved medication. 


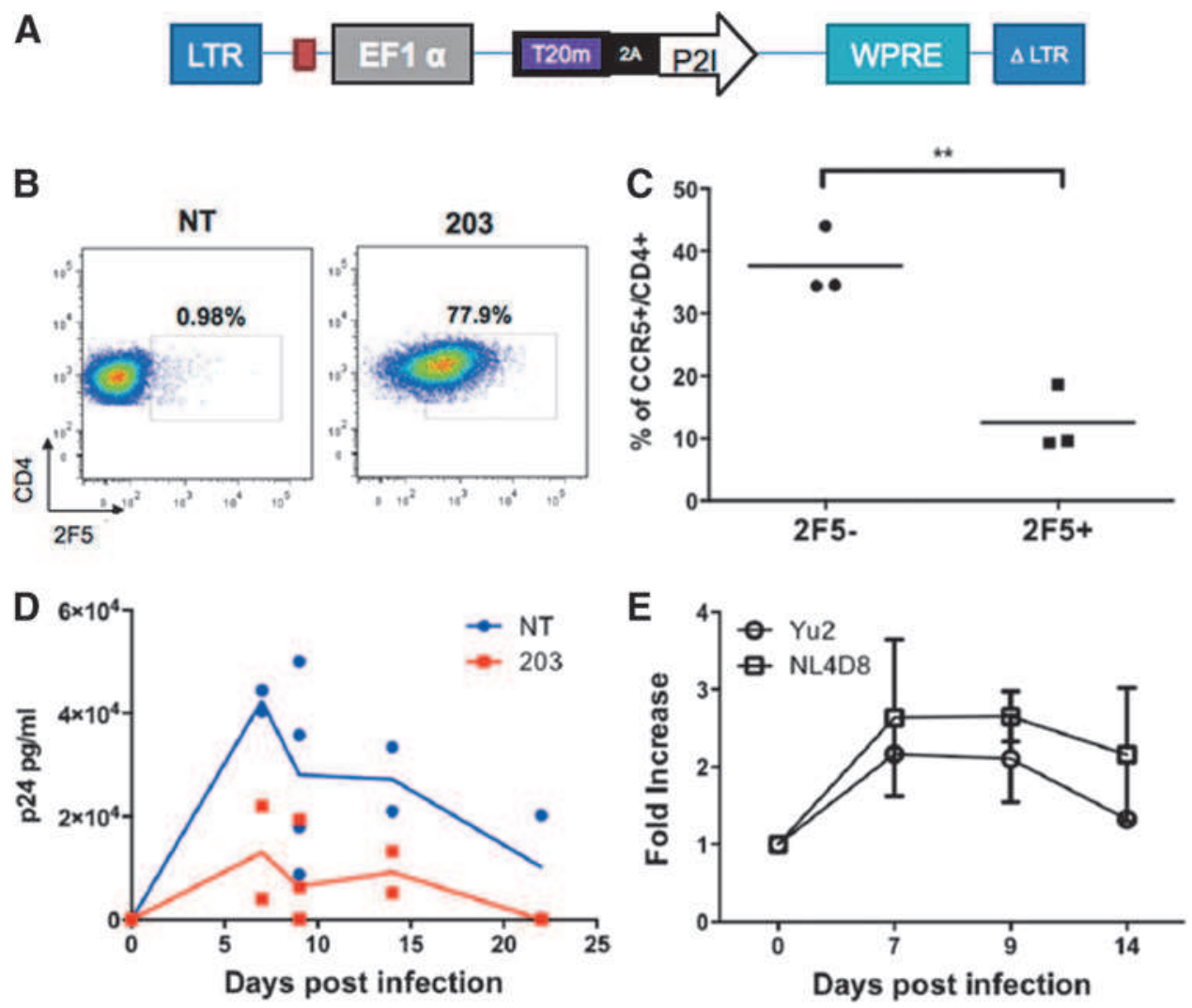

FIG. 5. Expression of C46 peptide and of P2-CCL5 intrakine efficiently protects primary human CD ${ }^{+} \mathrm{T}$ cells from infection. (A) A lentiviral vector coexpressing C46 peptide and P2-CCL5 intrakine was derived from the LvEF1 $\alpha$-GFP vector and is referred to as Lv203. (B) Expression levels of C46 peptide on anti-CD3/CD28-activated human peripheral blood mononuclear cells (PBMCs) 3 days after transduction with the Lv203 vector at an MOI of 40. Cells were stained for $\mathrm{C} 46$ expression with the $2 \mathrm{~F} 5$ monoclonal antibody $(\mathrm{mAb})$ and revealed with a secondary anti-human mAb labeled with phycoerythrin. NT, nontransduced. (C) Frequencies of CCR5 $5^{+}$cells gated in CD $4^{+} 2 \mathrm{~F} 5{ }^{+}$cells, as defined in (B), were determined by flow cytometry 22-23 days after transduction on CD3/CD28-activated PBMC cultures from three different donors in two independent experiments $(* * p<0.05)$. (D) HIV replication was monitored by p24 ELISA from the supernatant of $\mathrm{CD} 4{ }^{+} \mathrm{T}$ cells HIV-infected cultures, either mock transduced (NT, nontransduced) or transduced with the Lv203 vector after infection with either 0.5 or $25 \mathrm{ng}$ of p24 (NL4D8 R5-tropic virus) on $5 \times 10^{5}$ or $1 \times 10^{6}$ cells, respectively. Results shown are cumulative of two independent experiments with four unrelated healthy donors. (E) Selective advantage of transduced CD4 ${ }^{+} \mathrm{T}$ cells at an MOI of 20 on infection with Yu2 or NL4D8 virus (25 ng of p24), as indicated. Transduced cells were diluted with mock-transduced cells from the same donor to fix the frequencies of transduced cells at $20 \%$ before infection. The fold change represents the ratio of $2 \mathrm{~F} 5^{+}$cells determined by flow cytometry in HIV-infected versus noninfected cultures in one experiment with two healthy donors. Color images available online at www.liebertpub.com/hgtb

Despite the synergy between these two HIV inhibitors, we observed that gene-modified cells were not protected after challenge with high doses of the patient-derived HIV Yu2, in contrast to the molecular HIV construction NL4D8. The viral dose that a $\mathrm{CD} 4{ }^{+} \mathrm{T}$ cell might encounter in vivo is likely to be lower than the dose used in vitro, in our challenge experiments. Nevertheless, one might think of adding another therapeutic transgene to the vector to improve efficacy. The HIV-inhibiting integrase-binding domain (IBD) of lens epithelium-derived growth factor/p75 might represent a useful adjunct in our vector (De Rijck et al., 2006).

A major concern for antifusion therapeutic strategies is the rapid emergence of enfuvirtide-resistant variants (Greenberg and Cammack, 2004). However, it should be stressed that enfuvirtide-resistant HIV strains are still susceptible to membrane-bound C46 peptide (Egelhofer et al., 2004), showing that the resistance to $\mathrm{C} 46$ is not equivalent to that toward T20. Increasing local concentrations of T20 at the viral-cellular interface during fusion might be the cause of this improved efficacy. Of note is the fact that the $\mathrm{C} 46$ peptide is 10 amino acids longer than T20; that may also impact on the wider effect of C46 on T20-resistant HIV strains (Lohrengel et al., 2005). In any case, our strategy targeting essential steps of viral infection (fusion and attachment to CCR5) is likely to reduce the possible emergence of doubly-resistant escape variants that are generated during retrotranscription.

Another important feature needed for successful HIV gene therapy will be the selective advantage conferred to modified lymphocytes. We observed a selective advantage in vitro with a 2.1 -fold enrichment in $2 \mathrm{~F}^{+} \mathrm{CCR} 5^{\text {lo }}$ cells in HIV-challenged compared with noninfected cultures. Although this may appear modest, the selective advantage observed in vitro might be severely limited by indirect toxic 
effects linked to massive cell death in culture. A 2.2-fold in vivo selective advantage was reported for CCR5 and CXCR4 double-knockout cells (Didigu et al., 2014). A selective advantage has also been shown in humanized mice reconstituted with zinc finger-modified $\mathrm{CD} 34^{+}$progenitors (Holt et al., 2010) or with $\mathrm{CD} 4^{+} \mathrm{T}$ cells modified to express the C46 peptide (Kimpel et al., 2010). We are currently determining the selective advantage conferred by our vector to primary human $\mathrm{T}$ cells in vivo in advanced models of humanized mice. It will also be important to determine whether our protocol is able to significantly impact viral loads and $\mathrm{CD}^{+}{ }^{+} \mathrm{T}$ cell deletion.

In summary, our results functionally validate a new gene transfer vector combining two HIV entry inhibitors. Further work is needed to appreciate the impact of this approach in preclinical models of HIV gene therapy.

\section{Acknowledgments}

This work was funded by the French National Agency for AIDS Research (ANRS). N.P. is a recipient for a doctoral fellowship from the SIDAction/Fond de Dotation Pierre Bergé. A.B. is the recipient of a doctoral fellowship from the Ministère de la Recherche et de l'Enseignement Supérieur. The authors thank Dr. D. von Laer for the kind gift of the C46 cassette, Dr. A. Moris for the kind gift of reagents, and Prof. D. Klatzmann for initial support of this project.

\section{Author Disclosure Statement}

No competing financial interests exist.

\section{References}

Allers, K., Hütter, G., Hofmann, J., et al. (2011). Evidence for the cure of HIV infection by CCR $5 \Delta 32 / \Delta 32$ stem cell transplantation. Blood 117, 2791-2799.

Anderson, J., Li, M.-J., Palmer, B., et al. (2007). Safety and efficacy of a lentiviral vector containing three anti-HIV genes-CCR5 ribozyme, tat-rev siRNA, and TAR decoy-in SCID-hu mouse-derived T cells. Mol. Ther. 15, 1182-1188.

Deng, H., Liu, R., Ellmeier, W., et al. (1996). Identification of a major co-receptor for primary isolates of HIV-1 [see comments]. Nature 381, 661-666.

De Rijck, J., Vandekerckhove, L., Gijsbers, R., et al. (2006). Overexpression of the lens epithelium-derived growth factor/ p75 integrase binding domain inhibits human immunodeficiency virus replication. J. Virol. 80, 11498-11509.

Didigu, C.A., Wilen, C.B., Wang, J., et al. (2014). Simultaneous zinc-finger nuclease editing of the HIV coreceptors CCR5 and CXCR4 protects $\mathrm{CD} 4^{+}$T cells from HIV-1 infection. Blood 123, 61-69.

DiGiusto, D.L., Krishnan, A., Li, L., et al. (2010). RNA-based gene therapy for HIV with lentiviral vector-modified $\mathrm{CD} 34^{+}$: Cells in patients undergoing transplantation for AIDS-related lymphoma. Sci. Transl. Med. 2, 36ra43.

Doitsh, G., Galloway, N.L.K., Geng, X., et al. (2014). Cell death by pyroptosis drives CD4 T-cell depletion in HIV-1 infection. Nature 505, 509-514.

Dupuy, F.P., Mouly, E., Mesel-Lemoine, M., et al. (2005). Lentiviral transduction of human hematopoietic cells by
HIV-1- and SIV-based vectors containing a bicistronic cassette driven by various internal promoters. J. Gene Med. 7, 1158-1171.

Egelhofer, M., Brandenburg, G., Martinius, H., et al. (2004). Inhibition of human immunodeficiency virus type 1 entry in cells expressing gp41-derived peptides. J. Virol. 78, 568575.

Greenberg, M.L., and Cammack, N. (2004). Resistance to enfuvirtide, the first HIV fusion inhibitor. J. Antimicrob. Chemother. 54, 333-340.

Hartley, O., Dorgham, K., Perez-Bercoff, D., et al. (2003). Human immunodeficiency virus type 1 entry inhibitors selected on living cells from a library of phage chemokines. J. Virol. 77, 6637-6644.

Heredia, A., Gilliam, B., DeVico, A., et al. (2007). CCR5 density levels on primary CD4 $\mathrm{T}$ cells impact the replication and enfuvirtide susceptibility of R5 HIV-1. AIDS 21, 13171322.

Hildinger, M., Dittmar, M.T., Schult-Dietrich, P., et al. (2001). Membrane-anchored peptide inhibits human immunodeficiency virus entry. J. Virol. 75, 3038-3042.

Holt, N., Wang, J., Kim, K., et al. (2010). Human hematopoietic stem/progenitor cells modified by zinc-finger nucleases targeted to CCR5 control HIV-1 in vivo. Nat. Biotechnol. 28, 839-847.

Hutter, G., Nowak, D., Mossner, M., et al. (2009). Long-term control of HIV by CCR5 $\Delta 32 / \Delta 32$ stem-cell transplantation. N. Engl. J. Med. 360, 692-698.

Jin, H., Kagiampakis, I., Li, P., et al. (2010). Structural and functional studies of the potent anti-HIV chemokine variant P2-RANTES. Proteins 78, 295-308.

Judge, A.D., Sood, V., Shaw, J.R., et al. (2005). Sequencedependent stimulation of the mammalian innate immune response by synthetic siRNA. Nat. Biotechnol. 23, 457462.

Kimpel, J., Braun, S.E., Qiu, G., et al. (2010). Survival of the fittest: Positive selection of $\mathrm{CD}^{+} \mathrm{T}$ cells expressing a membrane-bound fusion inhibitor following HIV-1 infection. PLoS One 5, e12357.

Lohrengel, S., Hermann, F., Hagmann, I., et al. (2005). Determinants of human immunodeficiency virus type 1 resistance to membrane-anchored gp41-derived peptides. J. Virol. 79, 10237-10246.

Marodon, G., Warren, D., Filomio, M.C., et al. (1999). Productive infection of double negative T cells with HIV in vivo. Proc. Natl. Acad. Sci. U.S.A. 96, 11958-11963.

Marodon, G., Mouly, E., Blair, E.J., et al. (2003). Specific transgene expression in human and mouse $\mathrm{CD} 4{ }^{+}$cells using lentiviral vectors with regulatory sequences from the CD4 gene. Blood 101, 3416-3423.

Pattanayak, V., Ramirez, C.L., Joung, J.K., et al. (2011). Revealing off-target cleavage specificities of zinc-finger nucleases by in vitro selection. Nat. Methods 8, 765770.

Perez, E.E., Riley, J.L., Carroll, R.G., et al. (2005). Suppression of HIV-1 infection in primary CD4 T cells transduced with a self-inactivating lentiviral vector encoding a membrane expressed gp41-derived fusion inhibitor. Clin. Immunol. 115, 26-32.

Perez, E.E., Wang, J., Miller, J.C., et al. (2008). Establishment of HIV-1 resistance in $\mathrm{CD}^{+}{ }^{+} \mathrm{T}$ cells by genome editing using zinc-finger nucleases. Nat. Biotechnol. 26, 808-816. 
Peterson, C.W., Younan, P., Jerome, K.R., et al. (2013). Combinatorial anti-HIV gene therapy: Using a multipronged approach to reach beyond HAART. Gene Ther. 20, 695-702.

Qin, X.F., An, D.S., Chen, I.S., et al. (2003). Inhibiting HIV-1 infection in human $\mathrm{T}$ cells by lentiviral-mediated delivery of small interfering RNA against CCR5. Proc. Natl. Acad. Sci. U.S.A. 100, 183-188.

Robbins, M., Judge, A., Ambegia, E., et al. (2008). Misinterpreting the therapeutic effects of small interfering RNA caused by immune stimulation. Hum. Gene Ther. 19, 991999.

Swan, C.H., Buhler, B., Tschan, M.P., et al. (2006). T-cell protection and enrichment through lentiviral CCR5 intrabody gene delivery. Gene Ther. 13, 1480-1492.

Tebas, P., Stein, D., Tang, W.W., et al. (2014). Gene editing of CCR5 in autologous CD4 $\mathrm{T}$ cells of persons infected with HIV. N. Engl. J. Med. 370, 901-910.

Walker, J.E., Chen, R.X., McGee, J., et al. (2012). Generation of an HIV-1-resistant immune system with CD34 ${ }^{+}$: Hematopoietic stem cells transduced with a triplecombination anti-HIV lentiviral vector. J. Virol. 86, 57195729.

Yang, A.G., Bai, X., Huang, X.F., et al. (1997). Phenotypic knockout of HIV type 1 chemokine coreceptor CCR-5 by intrakines as potential therapeutic approach for HIV-1 infection. Proc. Natl. Acad. Sci. U.S.A. 94, 11567-11572.
Address correspondence to:

Dr. G. Marodon CIMI

INSERM U1135

CNRS ERL 8255

CERVI

$83 \mathrm{Bd}$ de l'Hôpital

F-75013 Paris

France

E-mail: gilles.marodon@upmc.fr

and

Prof. Guy Gorochov

CIMI

INSERM U1135

CERVI

83 Bd de l'Hôpital

F-75013 Paris

France

E-mail: guy.gorochov@upmc.fr

Received for publication April 2, 2014;

accepted after revision July 10, 2014.

Published online: July 21, 2014. 